\title{
CP Violations in Lepton Number Violation Processes and Neutrino Oscillations
}

\author{
K. MATSUDA, N. TAKEDA and T. FUKUYAMA \\ Department of Physics, \\ Ritsumeikan University, Kusatsu, \\ Shiga 525-8577, Japan \\ H. NISHIURA \\ Department of General Education, \\ Junior College of Osaka Institute of Technology, \\ Asahi-ku, Osaka 535-8585, Japan
}

(March 4, 2000)

\begin{abstract}
We examine the constraints on the MNS lepton mixing matrix from the present and future experimental data of the neutrino oscillation and lepton number violation processes. We introduce a graphical representation of the $C P$ violation phases which appear in the lepton number violation processes such as neutrinoless double beta decay, the $\mu^{-}-e^{+}$conversion, and the $\mathrm{K}$ decay, $K^{-} \rightarrow \pi^{+} \mu^{-} \mu^{-}$. Using this graphical representation, we derive the constraints on the $C P$ violation phases in the lepton sector.
\end{abstract}

PACS number(s): 14.60Pq, 11.30.Er, 23.40.Bw

Typeset using REVTEX 


\section{INTRODUCTION}

From the recent neutrino oscillation experiments it becomes affirmative that neutrinos have masses. The present and near future experiments enter into the stage of precision tests for masses and lepton mixing angles. In this situation the investigation of the $C P$ violation effects in the lepton sector has become more and more important. By taking account of the possible leptonic $C P$ violating phases for Majorana neutrinos, we have obtained the constraints on the lepton mixing angles from the neutrinoless double beta decay $\left((\beta \beta)_{0 \nu}\right)$ [1] [2], the $\mu^{-}-e^{+}$conversion and the $\mathrm{K}$ decay, $K^{-} \rightarrow \pi^{+} \mu^{-} \mu^{-}$[3]. In this paper, we propose graphical representations of the $C P$ violating phases which appear in those lepton number violating processes. By using those representations, we derive the allowed regions on the leptonic $C P$ violating phases from $(\beta \beta)_{0 \nu}$ without using any constraints on the mixing angles. We also try to determine the magnitude of the $C P$ violating phases by combining the constraints on the neutrino masses and mixing matrix elements from the recent Super Kamiokande atmospheric neutrino experiment [4], the solar neutrino experiment [5] [6] [7] [8] [9], the recent CHOOZ reactor experiment [10], and the future KamLAND reactor experiment [11] with those from the lepton number violating processes such as $(\beta \beta)_{0 \nu}$.

The amplitudes of those three lepton number violating processes are, in the absence of right-handed weak couplings, proportional to the "averaged" masses $\left\langle m_{\nu}\right\rangle_{e e},\left\langle m_{\nu}\right\rangle_{\mu e}$ and $\left\langle m_{\nu}\right\rangle_{\mu \mu}$. The "averaged" mass $\left\langle m_{\nu}\right\rangle_{e e}$ defined from $(\beta \beta)_{0 \nu}$ is given 12 by

$$
\left\langle m_{\nu}\right\rangle_{e e}=\left|\sum_{j=1}^{3} U_{e j}^{2} m_{j}\right| .
$$

Similarly, the "averaged" masses $\left\langle m_{\nu}\right\rangle_{\mu e}$ defined from $\mu^{-}-e^{+}$conversion and $\left\langle m_{\nu}\right\rangle_{\mu \mu}$ defined from the lepton number violating $\mathrm{K}$ decay, $K^{-} \rightarrow \pi^{+} \mu^{-} \mu^{-}$are given [13] [14] by

$$
\begin{aligned}
\left\langle m_{\nu}\right\rangle_{\mu e} & =\left|\sum_{j=1}^{3} U_{\mu j} U_{e j} m_{j}\right|, \\
\left\langle m_{\nu}\right\rangle_{\mu \mu} & =\left|\sum_{j=1}^{3} U_{\mu j}^{2} m_{j}\right|
\end{aligned}
$$

respectively. The $C P$ violating effects are included in the "averaged" masses $\left\langle m_{\nu}\right\rangle_{e e},\left\langle m_{\nu}\right\rangle_{\mu e}$ 
and $\left\langle m_{\nu}\right\rangle_{\mu \mu}$ defined in Eqs.(1.1) $\sim(1.3)$. Here $U_{a j}$ is the Maki-Nakagawa-Sakata (MNS) lefthanded lepton mixing matrix which combines the weak eigenstate neutrino $(a=e, \mu$ and $\tau)$ to the mass eigenstate neutrino with mass $m_{j}(j=1,2$ and 3$)$. The $U$ takes the following form in the standard representation [1]:

$$
U=\left(\begin{array}{ccc}
c_{1} c_{3} & s_{1} c_{3} e^{i \beta} & s_{3} e^{i(\rho-\phi)} \\
\left(-s_{1} c_{2}-c_{1} s_{2} s_{3} e^{i \phi}\right) e^{-i \beta} & c_{1} c_{2}-s_{1} s_{2} s_{3} e^{i \phi} & s_{2} c_{3} e^{i(\rho-\beta)} \\
\left(s_{1} s_{2}-c_{1} c_{2} s_{3} e^{i \phi}\right) e^{-i \rho} & \left(-c_{1} s_{2}-s_{1} c_{2} s_{3} e^{i \phi}\right) e^{-i(\rho-\beta)} & c_{2} c_{3}
\end{array}\right) .
$$

Here $c_{j}=\cos \theta_{j}, s_{j}=\sin \theta_{j}\left(\theta_{1}=\theta_{12}, \theta_{2}=\theta_{23}, \theta_{3}=\theta_{31}\right)$. Three $C P$ violating phases, $\beta$, $\rho$ and $\phi$ appear in $U$ for Majorana neutrinos [15]. In this paper we introduce the graphical representations of the complex masses $\sum_{j=1}^{3} U_{e j}^{2} m_{j}, \sum_{j=1}^{3} U_{\mu j} U_{e j} m_{j}$, and $\sum_{j=1}^{3} U_{\mu j}^{2} m_{j}$. Then, using these representations, we derive the constraints on the $C P$ violating phases which appear in the lepton number violating processes.

This article is organized as follows. In section 2 we introduce the graphical representations of the complex masses and the $C P$ violating phases. In section 3 we present constraints on the $C P$ violating phases from $(\beta \beta)_{0 \nu}$. Constraints from $(\beta \beta)_{0 \nu}$ and the neutrino oscillation experiments are discussed in section 4. Section 5 is devoted to summary.

\section{GRAPHICAL REPRESENTATIONS OF THE COMPLEX MASSES AND $C P$ VIOLATING PHASES}

We now rewrite the complex mass $\sum_{j=1}^{3} U_{e j}^{2} m_{j}$ by using the phase convention in Eq.(1.4) as

$$
\begin{aligned}
\sum_{j=1}^{3} U_{e j}^{2} m_{j} & =c_{1}^{2} c_{3}^{2} m_{1}+s_{1}^{2} c_{3}^{2} e^{2 i \beta} m_{2}+s_{3}^{2} e^{2 i(\rho-\phi)} m_{3} \\
& =\left|U_{e 1}\right|^{2} m_{1}+\left|U_{e 2}\right|^{2} e^{2 i \beta} m_{2}+\left|U_{e 3}\right|^{2} e^{2 i(\rho-\phi)} m_{3} \\
& \equiv\left|U_{e 1}\right|^{2} \widetilde{m_{1}}+\left|U_{e 2}\right|^{2} \widetilde{m_{2}}+\left|U_{e 3}\right|^{2} \widetilde{m_{3}}
\end{aligned}
$$

Here we have defined the complex masses $\widetilde{m_{i}}(i=1,2,3)$ by 


$$
\begin{aligned}
& \widetilde{m_{1}} \equiv m_{1} \\
& \widetilde{m_{2}} \equiv e^{2 i \beta} m_{2} \\
& \widetilde{m_{3}} \equiv e^{2 i \rho^{\prime}} m_{3}, \quad \rho^{\prime} \equiv \rho-\phi .
\end{aligned}
$$

We also rewrite the complex mass $\sum_{j=1}^{3} U_{\mu j} U_{e j} m_{j}$ by using the above $\widetilde{m}_{i}(i=1,2,3)$ as follows:

$$
\begin{aligned}
\sum_{j=1}^{3} U_{\mu j} U_{e j} m_{j} & =U_{e 1} U_{\mu 1} m_{1}+U_{e 2} U_{\mu 2} m_{2}+U_{e 3} U_{\mu 3} m_{3} \\
& =U_{e 1}^{*} U_{\mu 1} \widetilde{m}_{1}+U_{e 2}^{*} U_{\mu 2} \widetilde{m}_{2}+U_{e 3}^{*} U_{\mu 3} \widetilde{m}_{3} \\
& =U_{e 2}^{*} U_{\mu 2}\left(\widetilde{m}_{2}-\widetilde{m}_{1}\right)+U_{e 3}^{*} U_{\mu 3}\left(\widetilde{m}_{3}-\widetilde{m}_{1}\right)
\end{aligned}
$$

Here we have used the unitarity constraint that $\sum_{j=1}^{3} U_{e j}^{*} U_{\mu j}=0$. Furthermore, using $U_{\mu 1} \equiv$ $\left|U_{\mu 1}\right| e^{i\left(\varphi_{21}-\beta\right)}, U_{\mu 2} \equiv\left|U_{\mu 2}\right| e^{i \varphi_{22}}, U_{\mu 3}=\left|U_{\mu 3}\right| e^{i(\rho-\beta)}, U_{e 2}=\left|U_{e 2}\right| e^{i \beta}$ and $U_{e 3}=\left|U_{e 3}\right| e^{i(\rho-\beta)}$ with $\varphi_{21} \equiv \arg \left(-s_{1} c_{2}-c_{1} s_{2} s_{3} e^{i \phi}\right)$ and $\varphi_{22} \equiv \arg \left(c_{1} c_{2}-s_{1} s_{2} s_{3} e^{i \phi}\right)$, we obtain

$$
\begin{aligned}
\sum_{j=1}^{3} U_{\mu j} U_{e j} m_{j} & =e^{i(\phi-\beta)}\left(\left|U_{e 2}^{*} U_{\mu 2}\right| e^{i\left(\varphi_{22}-\phi\right)}\left(\widetilde{m}_{2}-\widetilde{m}_{1}\right)+\left|U_{e 3}^{*} U_{\mu 3}\right|\left(\widetilde{m}_{3}-\widetilde{m}_{1}\right)\right), \\
\sum_{j=1}^{3} U_{\mu j}^{2} m_{j} & =\left|U_{\mu 1}\right|^{2} e^{2 i\left(\varphi_{21}-\beta\right)} m_{1}+\left|U_{\mu 2}\right|^{2} e^{2 i \varphi_{22}} m_{2}+\left|U_{\mu 3}\right|^{2} e^{2 i(\rho-\beta)} m_{3} \\
& =e^{2 i\left(\varphi_{21}-\beta\right)}\left(\left|U_{\mu 1}\right|^{2} \widetilde{m}_{1}+\left|U_{\mu 2}\right|^{2} e^{2 i\left(\varphi_{22}-\varphi_{21}\right)} \widetilde{m}_{2}+\left|U_{\mu 3}\right|^{2} e^{2 i\left(\phi-\varphi_{21}\right)} \widetilde{m}_{3}\right) .
\end{aligned}
$$

Therefore, the $\left\langle m_{\nu}\right\rangle_{e e},\left\langle m_{\nu}\right\rangle_{\mu e}$, and $\left\langle m_{\nu}\right\rangle_{\mu \mu}$ defined in Eqs.(1.1) (1.3) are reexpressed by the absolute values of averaged complex masses as

$$
\begin{aligned}
\left\langle m_{\nu}\right\rangle_{e e} & =\left|M_{e e}\right|, \\
\left\langle m_{\nu}\right\rangle_{\mu e} & =\left|M_{\mu e}\right|, \\
\left\langle m_{\nu}\right\rangle_{\mu \mu} & =\left|M_{\mu \mu}\right| .
\end{aligned}
$$

Here the averaged complex masses $M_{e e}, M_{\mu e}$, and $M_{\mu \mu}$ are defined by

$$
\begin{aligned}
M_{e e} & \equiv\left|U_{e 1}\right|^{2} \widetilde{m_{1}}+\left|U_{e 2}\right|^{2} \widetilde{m_{2}}+\left|U_{e 3}\right|^{2} \widetilde{m_{3}} \\
M_{\mu e} & \equiv\left|U_{e 2}^{*} U_{\mu 2}\right| e^{i\left(\varphi_{22}-\phi\right)}\left(\widetilde{m}_{2}-\widetilde{m}_{1}\right)+\left|U_{e 3}^{*} U_{\mu 3}\right|\left(\widetilde{m}_{3}-\widetilde{m}_{1}\right), \\
M_{\mu \mu} & \equiv\left|U_{\mu 1}\right|^{2} \widetilde{m}_{1}+\left|U_{\mu 2}\right|^{2} e^{2 i\left(\varphi_{22}-\varphi_{21}\right)} \widetilde{m}_{2}+\left|U_{\mu 3}\right|^{2} e^{2 i\left(\phi-\varphi_{21}\right)} \widetilde{m}_{3} .
\end{aligned}
$$


Now let us introduce graphical representations of the complex value of the $M_{e e}, M_{\mu e}$ and $M_{\mu \mu}$ in a complex mass plane in order to investigate the magnitude of the $C P$ violating phases in them. The $M_{e e}$ is the "averaged" complex mass of the masses $\widetilde{m}_{i}(i=$ $1,2,3)$ weighted by three mixing elements $\left|U_{e j}\right|^{2}(j=1,2,3)$ with the unitarity constraint $\sum_{j=1}^{3}\left|U_{e j}\right|^{2}=1$. Therefore, the position of $M_{e e}$ in a complex mass plane is within the triangle formed by the three mass points $\widetilde{m_{i}}(i=1,2,3)$ if the magnitudes of $\left|U_{e j}\right|^{2}(j=1,2,3)$ are unknown, which is shown in Fig. 1(a-i). Hereafter we refer this triangle as the complex-mass triangle. This triangle is different from that defined by Fogli et al. [9] in the sense that ours incorporates the $C P$ violating phases and masses.

The three mixing elements $\left|U_{e j}\right|^{2}(j=1,2,3)$ indicate the division ratios for the three portions of each side of the triangle which are divided by the parallel lines to the side lines of the triangle passing through the $M_{e e}$. (Fig. 1(a-ii)). The $C P$ violating phases $2 \beta$ and $2 \rho^{\prime}$ represent the rotation angles of $\widetilde{m_{2}}$ and $\widetilde{m_{3}}$ around the origin, respectively.

Likewise, the constraints on the positions of $M_{\mu e}$ and $M_{\mu \mu}$ are depicted in Figs.1(b) and 1(c). The position of $M_{\mu e}$ is given as Fig. 1(b). The position of $M_{\mu \mu}$ in a complex mass plane is within the triangle formed by the three mass points $\widetilde{m_{1}}, e^{2 i\left(\varphi_{22}-\varphi_{21}\right)} \widetilde{m_{2}}$, and $e^{2 i\left(\phi-\varphi_{21}\right)} \widetilde{m}_{3}$ which is shown in Fig. 1(c).

\section{CONSTRAINTS ON THE $C P$ VIOLATING PHASES FROM $(\beta \beta)_{0 \nu}$}

Among the lepton number violation processes such as $(\beta \beta)_{0 \nu}$, the $\mu^{-}-e^{+}$conversion and the $\mathrm{K}$ decay, $K^{-} \rightarrow \pi^{+} \mu^{-} \mu^{-}$, the $(\beta \beta)_{0 \nu}$ gives us most restrictive constraints on the $C P$ violating phases. Therefore, hereafter we concentrate on the $(\beta \beta)_{0 \nu}$ and derive constraints

on the $C P$ violating phases from the experimental upper bound on $\left\langle m_{\nu}\right\rangle_{e e}$ (we denote it $\left\langle m_{\nu}\right\rangle_{\max }$, i.e., $\left.\left\langle m_{\nu}\right\rangle_{e e}<\left\langle m_{\nu}\right\rangle_{\max }\right)$. Since $\left\langle m_{\nu}\right\rangle_{e e}=\left|M_{e e}\right|$, the present experimental upper bound on $\left\langle m_{\nu}\right\rangle_{e e}$ obtained from the $(\beta \beta)_{0 \nu}$ forms the circle in the complex plane and this circle must include the point $M_{e e}$ inside of it. Namely, the allowed region for $M_{e e}$ is the intersection of the inside of the circle of radius $\left\langle m_{\nu}\right\rangle_{\max }$ around the origin and the inside of 
the complex-mass triangle which was discussed in section 2.

In the case of $m_{1}>\left\langle m_{\nu}\right\rangle_{\max }$, we can obtain the constraints on the $C P$ violating phases from the allowed region for $M_{e e}$ without using any constraints on the mixing elements $\left|U_{e j}\right|^{2}(j=1,2,3)$ as follows. In order to obtain the conditions for the allowed $M_{e e}$, it is more convenient to survey the forbidden regions for $M_{e e}$. It is easily understood from Fig 2(a) that the complex-mass triangle does not overlap with the circle $\left\langle m_{\nu}\right\rangle_{\max }$ only if the following conditions are satisfied for all $i$ and $j$.

$$
\left|\arg \left(\widetilde{m_{j}} / \widetilde{m}_{i}\right)\right|<\alpha_{i j}
$$

Here $\alpha_{i j}$ is defined by $\alpha_{i j} \equiv \cos ^{-1}\left(\left\langle m_{\nu}\right\rangle_{\max } / m_{i}\right)+\cos ^{-1}\left(\left\langle m_{\nu}\right\rangle_{\max } / m_{j}\right)$. Therefore, the allowed region for $M_{e e}$ is the area where, at least, one of the inequalities of Eq. (3.1) is violated. Since we have $\left|\arg \left(\widetilde{m_{2}} / \widetilde{m_{1}}\right)\right|=|2 \beta|,\left|\arg \left(\widetilde{m_{3}} / \widetilde{m_{1}}\right)\right|=\left|2 \rho^{\prime}\right|$, and $\left|\arg \left(\widetilde{m_{2}} / \widetilde{m_{3}}\right)\right|=\left|2 \beta-2 \rho^{\prime}\right|$, with $2 \beta$ and $2 \rho^{\prime}$ in the interval of $(-\pi, \pi)$, we find that Majorana $C P$ violating phases $\beta$ and $\rho^{\prime}$, must satisfy the following conditions:

$$
\alpha_{12}<|2 \beta| \quad \text { or } \quad \alpha_{13}<\left|2 \rho^{\prime}\right| \quad \text { or } \quad \alpha_{23}<\left|2 \rho^{\prime}-2 \beta\right| \text {. }
$$

The allowed region in the $2 \beta$ vs $2 \rho^{\prime}$ plane obtained from Eq.(3.2) is depicted in Fig. 2(b).

Eq.(3.2) is also useful in the case where the three neutrino masses are almost degenerate and $\left\langle m_{\nu}\right\rangle_{\max }<m_{1} \simeq m_{2} \simeq m_{3} \equiv m$. In this case, Eq.(3.2) reduces to

$$
\alpha<|2 \beta| \quad \text { or } \quad \alpha<\left|2 \rho^{\prime}\right| \quad \text { or } \quad \alpha<\left|2 \rho^{\prime}-2 \beta\right|
$$

with $\alpha \equiv 2 \cos ^{-1}\left(\left\langle m_{\nu}\right\rangle_{\max } / m\right)$ and the allowed region Fig. 2(b) to Fig. 2(c).

\section{CONSTRAINTS ON THE $C P$ VIOLATING PHASES FROM $(\beta \beta)_{0 \nu}$ AND THE NEUTRINO OSCILLATION EXPERIMENTS}

Now, we consider the constraints on $\left|U_{e j}\right|^{2}$ from the CHOOZ reactor experiment, the recent Super-Kamiokande atmospheric neutrino experiment, solar neutrino experiments and the future KamLAND reactor experiment. Then, by combining these constraints on $\left|U_{e j}\right|^{2}$ 
with one from $(\beta \beta)_{0 \nu}$, we derive the possible constraints on the $C P$ violating phases by using our graphical representation. In the following discussions we consider three cases for the neutrino mass hierarchy, i.e., case(A): two quasi-degenerate neutrino with $m_{1} \sim m_{2} \ll m_{3}$, case(B): two quasi-degenerate neutrino with $m_{1} \ll m_{2} \sim m_{3}$ and case(C): three quasidegenerate neutrino with $m_{1} \simeq m_{2} \simeq m_{3}=m$.

\section{A. two quasi-degenerate neutrino with $m_{1} \sim m_{2} \ll m_{3}$}

In the case, the oscillation probability for reactor neutrinos in the three-generation model, $P\left(\bar{\nu}_{e} \rightarrow \bar{\nu}_{e}\right)$ is given by

$$
\begin{aligned}
P\left(\bar{\nu}_{e} \rightarrow \bar{\nu}_{e}\right) & =1-4\left|U_{e 3}\right|^{2}\left(1-\left|U_{e 3}\right|^{2}\right) \sin ^{2}\left(\frac{\Delta m_{13}^{2} L}{4 E}\right) \\
& =1-4 s_{3}^{2} c_{3}^{2} \sin ^{2}\left(\frac{\Delta m_{13}^{2} L}{4 E}\right)
\end{aligned}
$$

if $\Delta m_{13}^{2} L /(4 E) \ll 1$. The present $\mathrm{CHOOZ}$ experiment gives a severe restriction on the mixing angle:

$$
\sin ^{2} 2 \theta \lesssim 0.1
$$

In this case, since $\theta=\theta_{3}$, we obtain

$$
0 \leq s_{3}^{2} \lesssim 0.026 \quad \text { or } \quad 0.97 \lesssim s_{3}^{2} \leq 1
$$

On the other hand, the oscillation probability for the atmospheric neutrinos, $P\left(\nu_{\mu} \rightarrow \nu_{\mu}\right)$ is

$$
\begin{aligned}
P\left(\nu_{\mu} \rightarrow \nu_{\mu}\right) & =1-4\left|U_{\mu 3}\right|^{2}\left(1-\left|U_{\mu 3}\right|^{2}\right) \sin ^{2}\left(\frac{\Delta m_{13}^{2} L}{4 E}\right) \\
& =1-4 c_{3}^{2} s_{2}^{2}\left(1-c_{3}^{2} s_{2}^{2}\right) \sin ^{2}\left(\frac{\Delta m_{13}^{2} L}{4 E}\right) .
\end{aligned}
$$

The atmospheric $\nu_{\mu}$ deficit in the Super Kamiokande experiment indicates that $0.8 \lesssim$ $4 c_{3}^{2} s_{2}^{2}\left(1-c_{3}^{2} s_{2}^{2}\right) \leq 1$, namely we obtain 


$$
\frac{0.28}{1-s_{3}^{2}} \lesssim s_{2}^{2} \lesssim \frac{0.72}{1-s_{3}^{2}}
$$

From these two constraints, Eqs.(4.3) and (4.5), we obtain

$$
0.28 \lesssim s_{2}^{2} \lesssim 0.74, \quad s_{3}^{2} \lesssim 0.026
$$

Eq.(4.6) imposes the restriction on $\left|U_{e 3}\right|^{2}$. Therefore, when combined with the allowed region in the complex mass plane discussed in the section 2 , the position of $M_{e e}$ in our graphical representation is restricted by the CHOOZ and Super Kamiokande experiments as shown in Fig. 3. We also have the constraints on the mixing angle from the solar neutrino experiments. They give several separate allowed regions for the position of $M_{e e}$ in our graphical representation as shown in Fig. 3. Whether the mixing angle for solar neutrinos is large or small can be determined by the future KamLAND reactor experiment [11]. The future KamLAND experiment will also lead to the constraint on the $\left|U_{e 1}\right|^{2}$ and $\left|U_{e 2}\right|^{2}$. Since the KamLAND experiment has the chance to observe a lower order mass difference, $\Delta m^{2} \sim 10^{-5} \mathrm{eV}^{2}$, we can't neglect the term depend on $\Delta m_{12}^{2}$ in $P\left(\bar{\nu}_{e} \rightarrow \bar{\nu}_{e}\right)$. So we rewrite Eq.(4.1) as follows:

$$
\begin{aligned}
P\left(\bar{\nu}_{e} \rightarrow \bar{\nu}_{e}\right) & =1-4\left[\frac{\left|U_{e 3}\right|^{2}\left(1-\left|U_{e 3}\right|^{2}\right)}{2}+\left|U_{e 1}\right|^{2}\left|U_{e 2}\right|^{2} \sin ^{2}\left(\frac{\Delta m_{12}^{2} L}{4 E}\right)\right] \\
& =1-\left[\frac{2 s_{3}^{2} c_{3}^{2}}{\sin ^{2}\left(\frac{\Delta m_{12}^{2} L}{4 E}\right)}+4 s_{1}^{2} c_{1}^{2} c_{3}^{4}\right] \sin ^{2}\left(\frac{\Delta m_{12}^{2} L}{4 E}\right) . \\
& \equiv 1-\Xi^{2} \sin ^{2}\left(\frac{\Delta m_{12}^{2} L}{4 E}\right) .
\end{aligned}
$$

Here we have used the following conditions,

$$
\sin ^{2}\left(\frac{\Delta m_{13}^{2} L}{4 E}\right) \sim \frac{1}{2}, \quad \sin ^{2}\left(\frac{\Delta m_{23}^{2} L}{4 E}\right) \sim \frac{1}{2},
$$

because of their frequent oscillations. Let us combine Eq.4.7) with the constraint given in Eq.(4.6) which is obtained from the CHOOZ and Super Kamiokande experiments. Then the KamLAND experiment will give the constraints on $\left|U_{e 1}\right|^{2}$ and $\left|U_{e 2}\right|^{2}$, which will restrict the allowed region for the position of $M_{e e}$ as shown in Fig. 4. 
Now, with use of our graphical representation, we proceed to discuss the main subject in this paper: If we have non zero value of $\left\langle m_{\nu}\right\rangle_{e e}$, how can we determine the magnitude of Majorana $C P$ phases $\beta$ or $\rho^{\prime}$ ?

First we discuss the simple case in which $\left|U_{e 3}\right|^{2}$ is approximately zero and the large mixing angle solution(LMA), $0.2 \lesssim\left|U_{e 1}\right|^{2} \lesssim 0.8$, is adopted for the solar neutrino problem. In this case, we have

$$
\begin{aligned}
\left\langle m_{\nu}\right\rangle_{e e} & \left.\simeq|| U_{e 1}\right|^{2} \widetilde{m}_{1}+\left|U_{e 2}\right|^{2} \widetilde{m}_{2} \mid \\
& \left.\equiv|| U_{e 1}\right|^{2} \widetilde{m}_{1}+\left(1-\left|U_{e 1}\right|^{2}\right) \widetilde{m}_{2} \mid .
\end{aligned}
$$

Given the values of $\left\langle m_{\nu}\right\rangle_{e e}, m_{1}, m_{2},\left|U_{e 1}\right|^{2}$ and $\left|U_{e 2}\right|^{2}$, the $C P$ violating phase $\beta$ is easily obtained from the graphical representation of Eq.(4.9). It goes from Fig.1(a-ii) that the complex-mass triangle gets degenerate to a straight line $\widetilde{m_{1} \widetilde{m_{2}}}$ for $\left|U_{e 3}\right|^{2}=0$ case and that the position of $M_{e e}=\left|U_{e 1}\right|^{2} \widetilde{m_{1}}+\left|U_{e 2}\right|^{2} \widetilde{m_{2}}$ moves along the circle with a radius of $\left|U_{e 2}\right|^{2} m_{2}\left(=\left(1-\left|U_{e 1}\right|^{2}\right) m_{2}\right)$ from the point $\left|U_{e 1}\right|^{2} m_{1}$ for changing $\beta$. On the other hand, the measurement of the $\left\langle m_{\nu}\right\rangle_{e e}$ restricts $M_{e e}$ on the circle with a radius of $\left\langle m_{\nu}\right\rangle_{e e}$ from the origin. Therefore, the $\beta$ is determined by the intersection of the above two circles as shown in Fig.5. Applying the cosine formula to $\triangle O A B$ in Fig.5, we find

$$
\left\langle m_{\nu}\right\rangle^{2}=\left|U_{e 1}\right|^{4} m_{1}^{2}+\left|U_{e 2}\right|^{4} m_{2}^{2}+2\left|U_{e 1}\right|^{2}\left|U_{e 2}\right|^{2} m_{1} m_{2} \cos 2 \beta .
$$

Therefore, we obtain

$$
\cos 2 \beta=\frac{\left\langle m_{\nu}\right\rangle^{2}-\left|U_{e 1}\right|^{4} m_{1}^{2}-\left|U_{e 2}\right|^{4} m_{2}^{2}}{2\left|U_{e 1}\right|^{2}\left|U_{e 2}\right|^{2} m_{1} m_{2}} .
$$

It goes from Eq.(4.11) with the use of $-1 \leq \cos 2 \beta \leq 1$ that $\left\langle m_{\nu}\right\rangle_{e e}$ has the lower and upper limits as $\left\langle m_{\nu}\right\rangle_{\text {lower }} \leq\left\langle m_{\nu}\right\rangle_{e e} \leq\left\langle m_{\nu}\right\rangle_{\text {upper }}$, which is shown in Fig.6 with the definitions of

$$
\begin{gathered}
\left\langle m_{\nu}\right\rangle_{\text {lower }}=\left.\left|m_{1}-\right| U_{e 2}\right|^{2}\left(m_{1}+m_{2}\right) \mid \\
\left\langle m_{\nu}\right\rangle_{\text {upper }}=m_{1}+\left|U_{e 2}\right|^{2}\left(m_{2}-m_{1}\right) .
\end{gathered}
$$

On the other hand, for the case where $\left|U_{e 3}\right|^{2} \simeq 0$ and the small mixing angle solution(SMA) is adopted for the solar neutrino problem, i.e., $\theta_{1}=0$ or $\pi / 2$, we can not obtain any 
information about $\beta$, since we have $\left\langle m_{\nu}\right\rangle_{e e}=\left|m_{1}\right|=m_{1}$ for $\theta_{1}=0$ or $\left\langle m_{\nu}\right\rangle_{e e}=\left|e^{2 i \beta} m_{2}\right|=m_{2}$ for $\theta_{1}=\pi / 2$.

Second we consider the case where $\left|U_{e 3}\right|^{2} \neq 0$ and the LMA solution, $0.2 \lesssim\left|U_{e 1}\right|^{2} \lesssim 0.8$, is adopted for the solar neutrino problem. In this case, we have

$$
M_{e e}-\left|U_{e 3}\right| \widetilde{m_{3}}=\left|U_{e 1}\right|^{2} m_{1}+\left|U_{e 2}\right|^{2} \widetilde{m_{2}}
$$

The graphical representation of Eq.(4.13) is shown in Fig.7. In Fig.7(a) we consider the case in which the circle of radius $\left|U_{e 2}\right|^{2} m_{2}$ around the point $\left(\left|U_{e 1}\right|^{2} m_{1}, 0\right)$ (which we refer as $A$ or $\overrightarrow{O A})$ intersects with the circles of radius $\left\langle m_{\nu}\right\rangle_{e e} \pm\left|U_{e 3}\right|^{2} m_{3}$ around the origin at the points $B_{1}$ and $B_{2}$. We find that $2 \beta$ is ranging from the argument of $\overrightarrow{A B_{1}}$ to that of $\overrightarrow{A B_{2}}$ as seen in Fig.7(a). The relation between $\beta$ and $\rho^{\prime}$ is also derived from Eq.(4.13): For fixed $2 \beta$, the $\rho^{\prime}$ has two solution $\rho_{1}^{\prime}$ and $\rho_{2}^{\prime}$ which are determined by the points $C_{1}$ and $C_{2}$ as shown in Fig.7(b). Here the $C_{1}$ and $C_{2}$ are the intersections of the circle of radius $\left|U_{e 3}\right|^{2} m_{3}$ around the point $\left|U_{e 1}\right|^{2} \widetilde{m_{1}}+\left|U_{e 2}\right|^{2} \widetilde{m_{2}}$ (which we refer as $B$ ) with the circle of radius $\left\langle m_{\nu}\right\rangle_{e e}$ around the origin since $\overrightarrow{O A}+\overrightarrow{A B}+\overrightarrow{B C}=M_{e e}$ from Eq.(4.13). Thus we obtain the relation between $\beta$ and $\rho^{\prime}$. We depict this relation in Fig. 8. The other cases may occur but they can be treated analogously.

\section{B. two quasi-degenerate neutrino with $m_{1} \ll m_{2} \sim m_{3}$}

In this case, the CHOOZ experiment and the atmospheric neutrino deficit experiment indicates $\left|U_{e 1}\right|^{2} \sim 0$ as is seen in Fig. 9. Therefore, we can discuss this case with the same way as the case(A) only by replacing $m_{1},\left|U_{e 1}\right|^{2}$, and $\beta$ with $m_{3},\left|U_{e 3}\right|^{2}$, and $\beta-\rho^{\prime}$, respectively.

\section{C. three quasi-degenerate neutrino with $m_{1} \simeq m_{2} \simeq m_{3}=m$}

We assume that all three neutrino masses are almost degenerate, then we have

$$
M_{e e}=m\left(\left|U_{e 1}\right|^{2}+\left|U_{e 2}\right|^{2} e^{2 i \beta}+\left|U_{e 3}\right|^{2} e^{2 i \rho^{\prime}}\right) .
$$


The constraints on the $C P$ violating phases from this $M_{e e}$ is obtained from the similar discussions as in Fig. 7 with only taking $m_{1}=m_{2}=m_{3}=m$ in it. It should be noted that for the case where $\Delta m_{12}^{2} \ll \Delta m_{13}^{2}$ and $\left|U_{e 3}\right|^{2}$ is approximately zero, we find

$$
\begin{aligned}
\sin ^{2} \beta & =\frac{m^{2}-\left\langle m_{\nu}\right\rangle_{e e}^{2}}{4\left|U_{e 1}\right|^{2}\left(1-\left|U_{e 1}\right|^{2}\right) m^{2}} \\
& =\frac{m^{2}-\left\langle m_{\nu}\right\rangle_{e e}^{2}}{4 s_{1}^{2}\left(1-s_{1}^{2}\right) m^{2}} .
\end{aligned}
$$

which is the same result as one obtained from Eq.(4.11) with replacing $m_{i}(i=1,2,3)$ with $m$. We find from Eq.(4.15) that the following lower limit of $\sin ^{2} \beta$ is obtained for the large mixing angle solution(LMA), $0.2 \lesssim\left|U_{e 1}\right|^{2}=s_{1}^{2} \lesssim 0.8$, of the solar neutrino problem,

$$
\sin ^{2} \beta \geq 1-\left(\frac{\left\langle m_{\nu}\right\rangle_{e e}}{m}\right)^{2},
$$

where the lower limit is realized at $s_{1}^{2}=0.5$.

\section{SUMMARY}

We have introduced graphical representations of the complex masses, $M_{e e}, M_{\mu e}$ and $M_{\mu \mu}$ whose absolute magnitudes are experimentally observable "averaged" masses, $\left\langle m_{\nu}\right\rangle_{e e}$, $\left\langle m_{\nu}\right\rangle_{\mu e}$ and $\left\langle m_{\nu}\right\rangle_{\mu \mu}$ of the lepton number violation processes such as neutrinoless double beta decay, the $\mu^{-}-e^{+}$conversion and the $\mathrm{K}$ decay, $K^{-} \rightarrow \pi^{+} \mu^{-} \mu^{-}$, respectively. By using those graphical representations, we have investigated how to determine the magnitude of the $C P$ violating phases from the analysis of the neutrinoless double beta decay. First we have discussed without using any constraint on the mixing elements $\left|U_{e j}\right|^{2}(j=1,2,3)$ and obtained the constraints on the Majorana $C P$ violating phases, Eqs.(3.2) and (3.3) if $\left\langle m_{\nu}\right\rangle_{\max }<m_{1}$, from which the allowed region in the $2 \beta$ vs $2 \rho^{\prime}$ plane have been derived and shown in Fig. 2. Of course, we have no constraint on the Majorana $C P$ phases if $\left.\left\langle m_{\nu}\right\rangle_{\max }\right\rangle$ $m_{1}$. Still, Eq.(3.3) is useful if the three neutrino masses are almost degenerate and $\left\langle m_{\nu}\right\rangle_{\max }<$ $m_{1} \simeq m_{2} \simeq m_{3}=m$. Next by using the constraints on the mixing elements $\left|U_{e j}\right|^{2}(j=1,2,3)$

obtained from the recent Super Kamiokande atmospheric neutrino experiment, the solar 
neutrino experiment, the recent $\mathrm{CHOOZ}$ reactor experiment, and the future KamLAND reactor experiment, we have further discussed the possible constraints on the Majorana $C P$ violating phases for three cases for the neutrino mass hierarchy, i.e., case(A): two quasidegenerate neutrino with $m_{1} \sim m_{2} \ll m_{3}$, case(B): two quasi-degenerate neutrino with $m_{1} \ll m_{2} \sim m_{3}$ and case $(\mathrm{C})$ : three quasi-degenerate neutrino with $m_{1} \simeq m_{2} \simeq m_{3}=m$. In the case(A), we have obtained the expression of $\cos 2 \beta$, Eq.(4.11), in terms of $m_{1}, m_{2},\left\langle m_{\nu}\right\rangle_{e e}$, $\left|U_{e 1}\right|^{2}$, and $\left|U_{e 2}\right|^{2}$ for the simple case where $\left|U_{e 3}\right|^{2} \simeq 0$ with the use of the large mixing angle solution(LMA) for the solar neutrino problem. The $\left\langle m_{\nu}\right\rangle_{e e}$ vs $\cos 2 \beta$ relation is shown in Fig. 5. Using $-1 \leq \cos 2 \beta \leq 1$, we have found that the $\left\langle m_{\nu}\right\rangle_{e e}$ has the lower and upper limits as given in Eq.(4.12). We have also obtained the relation between $2 \beta$ and $2 \rho^{\prime}$ for $\left|U_{e 3}\right|^{2} \neq 0$ case which is shown in Figs. 7 and 8. We can discuss the case(B) using the same way as the case(A) by replacing $m_{1},\left|U_{e 1}\right|^{2}$, and $\beta$ with $m_{3},\left|U_{e 3}\right|^{2}$, and $\beta-\rho^{\prime}$, respectively. In the case(C), we have obtained the expression of $\sin ^{2} \beta$ given in Eq.(4.15) for the simple case where $\Delta m_{12}^{2} \ll \Delta m_{13}^{2}$ and $\left|U_{e 3}\right|^{2} \simeq 0$ with the use of the LMA solution for the solar

neutrino problem. From this relation we have found that the lower limit of $\sin ^{2} \beta$ is given by $\sin ^{2} \beta \geq 1-\left(\left\langle m_{\nu}\right\rangle_{e e} / m\right)^{2}$ for the LMA solution.

\section{Acknowledgement}

We are greatly indebted to O.Yasuda for useful discussions. 


\section{REFERENCES}

[1] T. Fukuyama, K. Matsuda, and H. Nishiura, Phys. Rev. D57, 5844 (1998).

[2] T. Fukuyama, K. Matsuda, and H. Nishiura, Mod. Phys. Lett. A13, 2279 (1998).

[3] H. Nishiura, K. Matsuda, and T. Fukuyama, Mod. Phys. Lett. A14, 433 (1999).

[4] T. Kajita, talk at Neutrino '98 (Takayama, Japan, June 1998).

[5] K.S. Hirata et al., Phys. Rev. Lett. 63, 6 (1989); N. Hata, S. Bludman, and P. Langacker, Phys. Rev. D49, 3622 (1994); N. Hata and P. Langacker, Phys. Rev. D50, 632 (1994).

[6] R. Davis Jr., Proc. of the 6'th International Workshop on Neutrino Telescope, Venezia, March 1994, edited by M.Baldo Ceolin.

[7] P. Anselmann et al., Phys. Lett. B285, 376 (1992), ibid B357, 237 (1995).

[8] A.I. Abazov et al., Phys. Rev. Lett. 67, 3332 (1991); J.N. Abdurashitov et al., Phys. Lett. B328, 234 (1994).

[9] G.L. Fogli, E. Lisi, and D. Montanino, Phys. Rev. D54, 2048 (1996).

[10] M. Apollonio et al, hep-ex/9907037 and Phys. Lett. B338, 383 (1998).

[11] G. GRATTA, hep-ex/9905011.

[12] M. Doi, T. Kotani, H. Nishiura, K. Okuda, and E. Takasugi, Phys. Lett. 102B, 323 (1981); M. Doi, T. Kotani, and E. Takasugi, Prog. Theor. Phys. Suppl. 83, 1 (1985); W.C. Haxton and G.J. Stophenson Jr., Prog. Part. Nucl. Phys. 12, 409 (1984).

[13] M. Doi, T. Kotani, and E. Takasugi, Prog. Theor. Phys. Suppl. 83 (1985).

[14] H. Nishiura, Phys. Lett. 157B, 442 (1985).

[15] S.M. Bilenky, J. Hosek, and S.T. Petcov, Phys. Lett. 94B, 495 (1980); M. Doi, T. Kotani, H. Nishiura, K. Okuda, and E. Takasugi, Phys. Lett. 102B, 323 (1981). 
[16] B. Achkar et al., Nucl. Phys. B434, 503 (1995).

[17] K. Winter, Nucl. Phys. B38 (Proc. Suppl.), 211 (1995).

[18] L. DiLella, Nucl. Phys. B31 (Proc. Suppl.), 319 (1993).

[19] Particle Data Group, R.M. Barnet et al., Phys. Rev. D54, 1 (1997). 

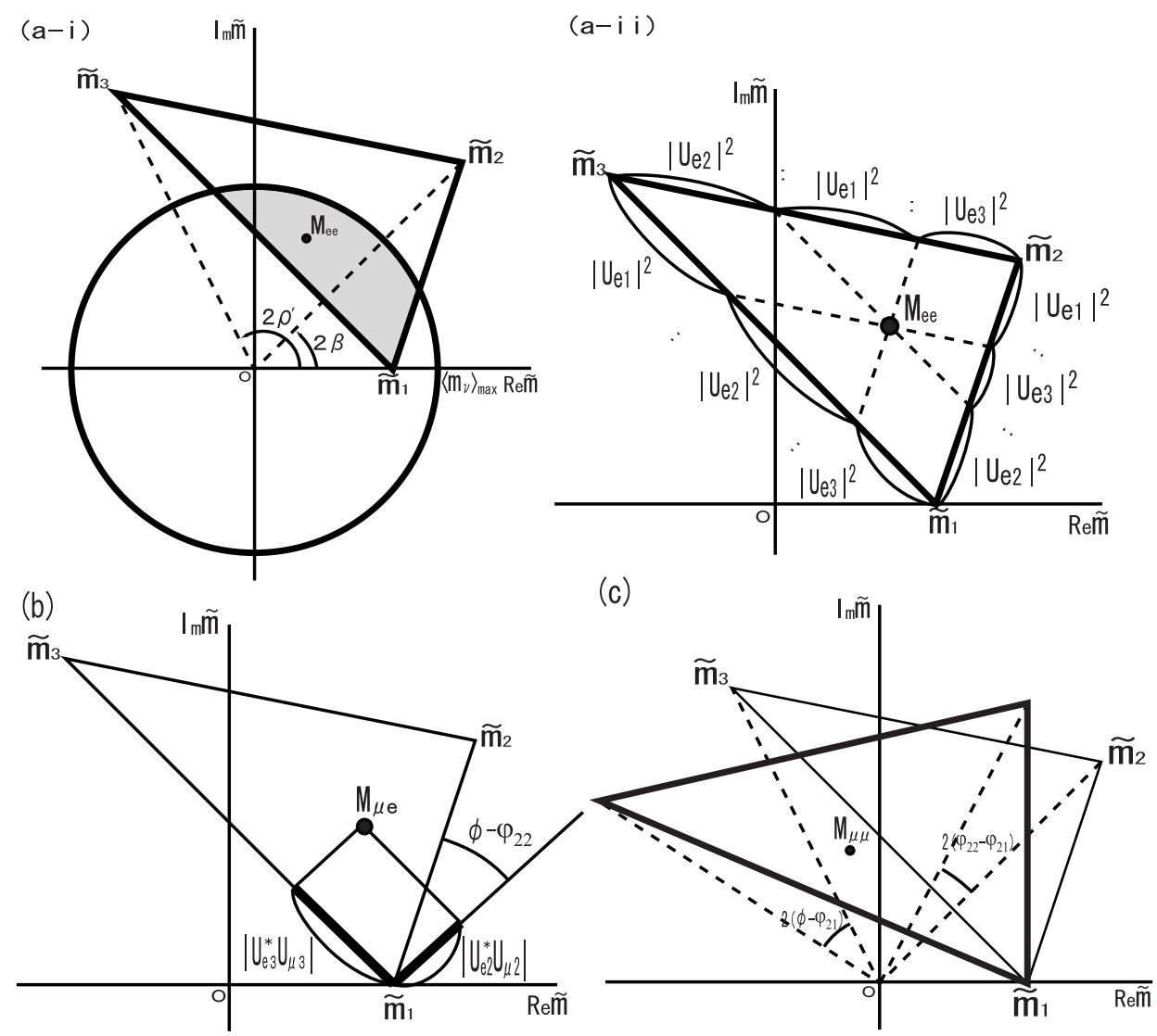

FIG.1 Graphical representations of the $C P$ violating phases and the complex masses $M_{e e}$, $M_{\mu e}$ and $M_{\mu \mu}$ defined in Eqs.(2.6)-(2.8). (a-i) The complex-mass triangle for $M_{e e}$ is formed by the three points $\widetilde{m}_{i}(i=1,2,3)$ defined in Eqs.(2.2a)-(2.2G). The allowed position of $M_{e e}$ is in the intersection (shaded area) of the inside of this triangle and the inside of the circle of radius $\left\langle m_{\nu}\right\rangle_{\max }$ around the origin. (a-ii) The relations between the position of $M_{e e}$ and $U_{e i}(i=1,2,3)$ components of MNS mixing matrix. (b) The position of $M_{\mu e}$. The position of $M_{\mu e}$ is at the vertex of the parallelogram of which the other vertexes are at $\widetilde{m_{1}}$, $\left|U_{e 2}^{*} U_{\mu 2}\right| e^{2 i\left(\varphi_{22}-\varphi_{21}\right)}\left(\widetilde{m_{2}}-\widetilde{m_{1}}\right)$, and $\left|U_{e 3}^{*} U_{\mu 3}\right| e^{2 i\left(\phi-\varphi_{21}\right)}\left(\widetilde{m}_{3}-\widetilde{m_{1}}\right)$. Namely, rotate $\widetilde{m_{2}}$ clockwise by $\phi-\varphi_{22}$ around $\widetilde{m_{1}}$ and scale down by $\left|U_{e 2}^{*} U_{\mu 2}\right|$. From this point extend the line parallel to the side of $\widetilde{m_{1}} \widetilde{m_{3}}$ by $\left|U_{e 3}^{*} U_{\mu 3}\right|\left|\widetilde{m_{3}}-\widetilde{m_{1}}\right|$, then we obtain the position of $M_{\mu e}$. (c) The complex-mass triangle for $M_{\mu \mu}$ (thick lines). The allowed position of $M_{\mu \mu}$ is within the triangle formed by the three points $\widetilde{m_{1}}, e^{2 i\left(\varphi_{22}-\varphi_{21}\right)} \widetilde{m_{2}}$, and $e^{2 i\left(\phi-\varphi_{21}\right)} \widetilde{m}_{3}$. 

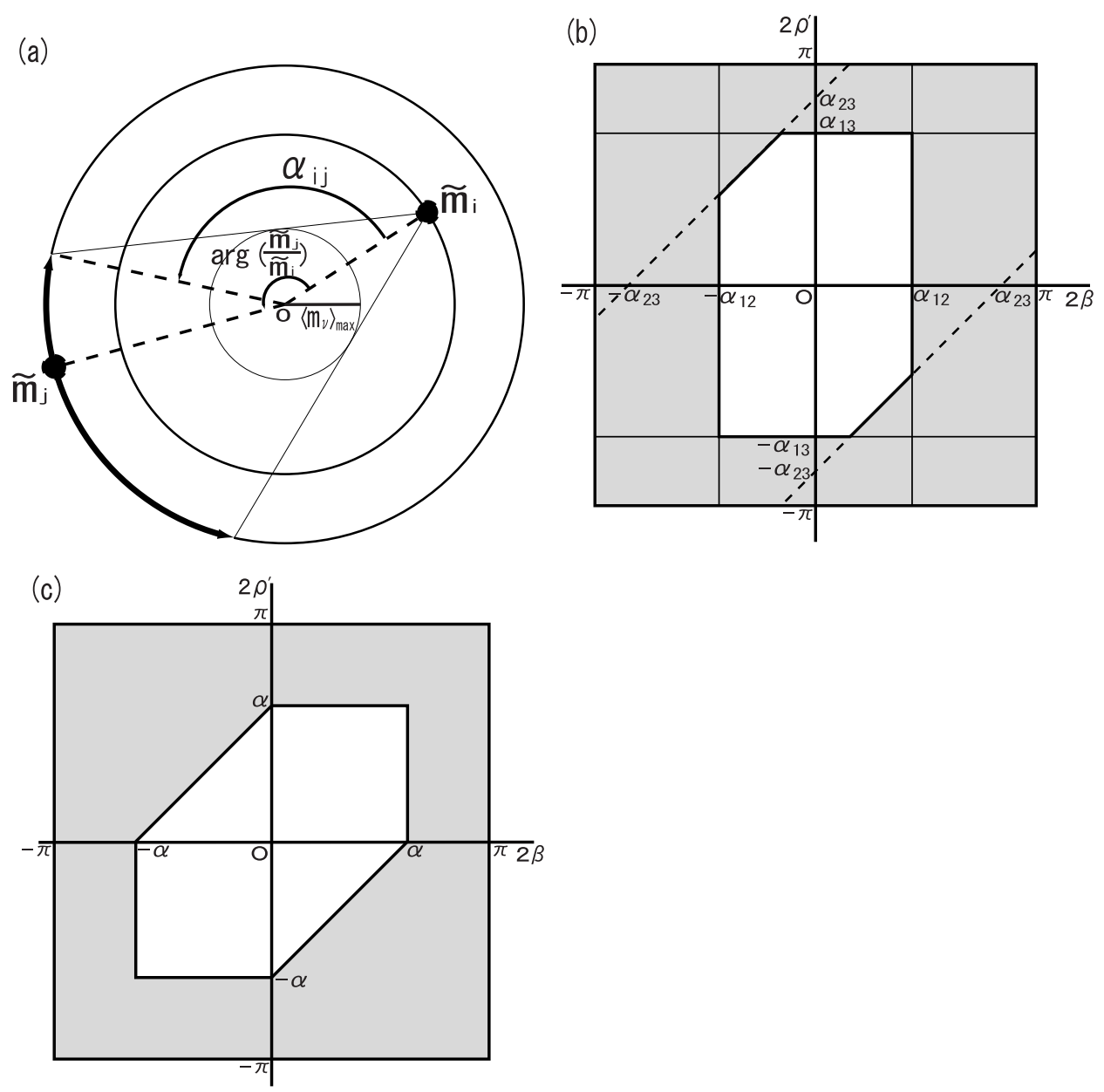

FIG.2 The restrictions of $\mathrm{CP}$ violating phases $2 \beta$ and $2 \rho^{\prime}$ from $(\beta \beta)_{0 \nu}$ with arguments independent of $\left|U_{e j}\right|^{2}$. (a) The allowed region of $M_{e e}$ is inside of the complex-mass triangle overlapped with the inside of the circle of radius $\left\langle m_{\nu}\right\rangle_{\max }$. The case where the conditions $\left|\alpha_{i j}\right|>\left|\arg \left(\widetilde{m_{j}} / \widetilde{m_{i}}\right)\right|$ are satisfied for all $i$ and $j$ is excluded since the triangle and the circle can not overlap each other. Here we define $\alpha_{i j} \equiv \cos ^{-1}\left(\left\langle m_{\nu}\right\rangle_{\max } / m_{i}\right)+\cos ^{-1}\left(\left\langle m_{\nu}\right\rangle_{\max } / m_{j}\right)$, $\left|\arg \left(\widetilde{m_{2}} / \widetilde{m_{1}}\right)\right| \equiv|2 \beta|<\pi,\left|\arg \left(\widetilde{m_{3}} / \widetilde{m_{1}}\right)\right| \equiv\left|2 \rho^{\prime}\right|<\pi$, and, therefore, $\left|\arg \left(\widetilde{m_{2}} / \widetilde{m_{3}}\right)\right| \equiv$ $\left|2 \beta-2 \rho^{\prime}\right|<2 \pi$. (b) The allowed region (shaded area) in the $2 \beta$ vs $2 \rho^{\prime}$ plane for $\left\langle m_{\nu}\right\rangle_{\max }<m_{1}<m_{2}<m_{3}$ case. (c) The special case of Fig.2 (b) for the case in which three neutrinos have almost degenerate masses with $\left\langle m_{\nu}\right\rangle_{\max }<m_{1} \simeq m_{2} \simeq m_{3}$. Here $\alpha \equiv 2 \cos ^{-1}\left(\left\langle m_{\nu}\right\rangle_{\max } / m\right)$. 


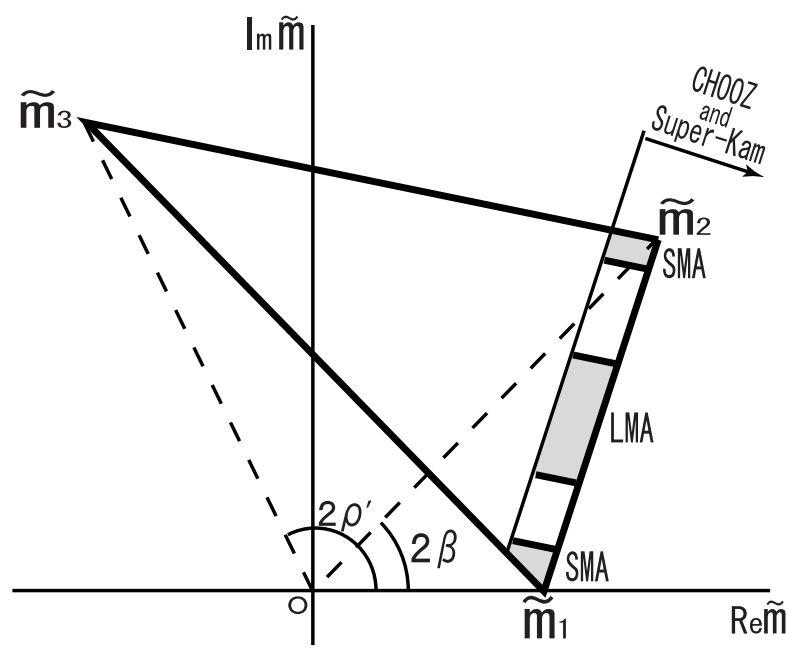

FIG.3 The allowed region (shaded area) of $M_{e e}$ for the case (A) from the CHOOZ, the atmospheric $\nu_{\mu}$ deficit and the solar neutrino experiments. The CHOOZ and the atmospheric $\nu_{\mu}$ deficit experiments restrict the position of $M_{e e}$ in the neighborhood of the side $\widetilde{m_{1}} \widetilde{m_{2}}$. The large mixing angle (LMA) and the small mixing angle (SMA) solutions for the solar neutrino problem give separate allowed regions. 

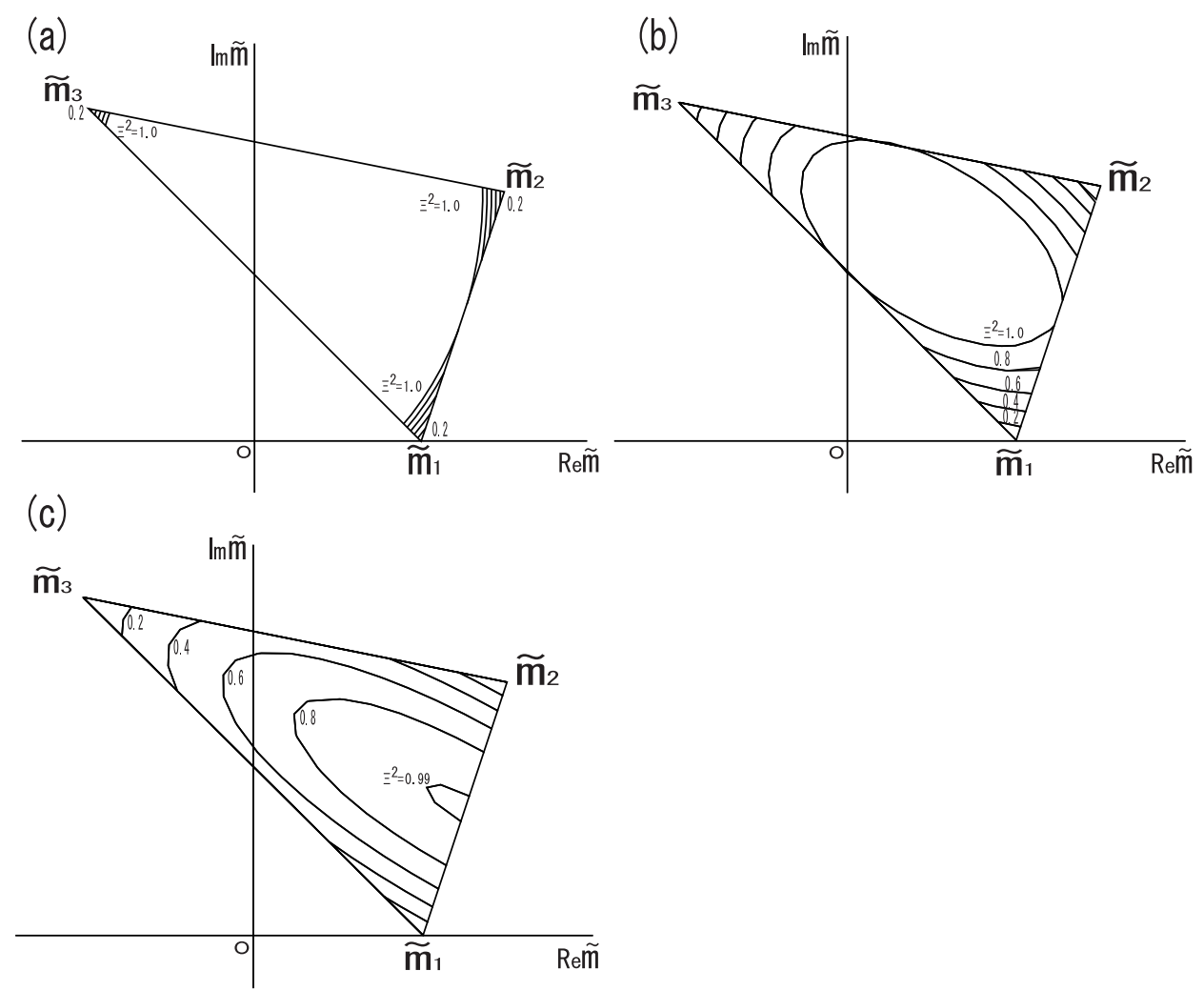

FIG.4 Constraints from KamLAND experiments. The contours of $\mathrm{P}\left(\overline{\nu_{e}} \rightarrow \overline{\nu_{e}}\right)$ in our complex-mass triangle. ( $\Xi$ is defined in Eq.(4.7).) They are plotted at the interval of 0.2 of $\Xi^{2}$ for the typical values of $\sin ^{2} \frac{\Delta m_{12}^{2}}{4 E} L$. (a); $\sin ^{2} \frac{\Delta m_{12}^{2}}{4 E} L=0.1$. (b); $\sin ^{2} \frac{\Delta m_{12}^{2}}{4 E} L=0.5$. (c); $\sin ^{2} \frac{\Delta m_{12}^{2}}{4 E} L=1$.

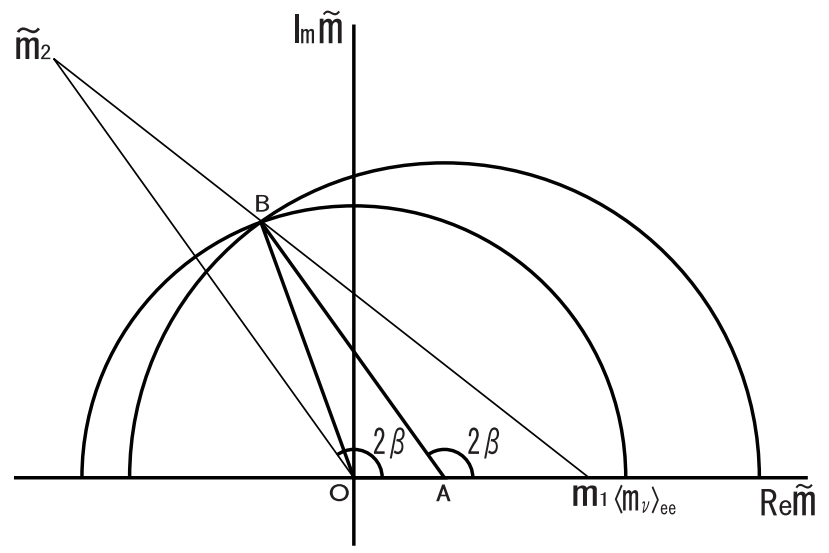

FIG.5 The determination of $\beta$ for $\left|U_{e 3}\right|^{2}=0$ in the case(A). The $\beta$ is determined from the point $B$ which is the intersection of two circles; the circle of radius $\left\langle m_{\nu}\right\rangle_{e e}$ around the origin 
and that of radius of $\left|U_{e 2}\right|^{2} m_{2}$ around $\left(\left|U_{e 1}\right|^{2} m_{1}, 0\right)$ (which we refer as $A$ ). The line $A B$ is parallel to the line $O \widetilde{m_{2}}$. Here $|O A|=\left|U_{e 1}\right|^{2} m_{1},|O B|=\left\langle m_{\nu}\right\rangle_{e e},|A B|=\left|U_{e 2}\right|^{2} m_{2}$.

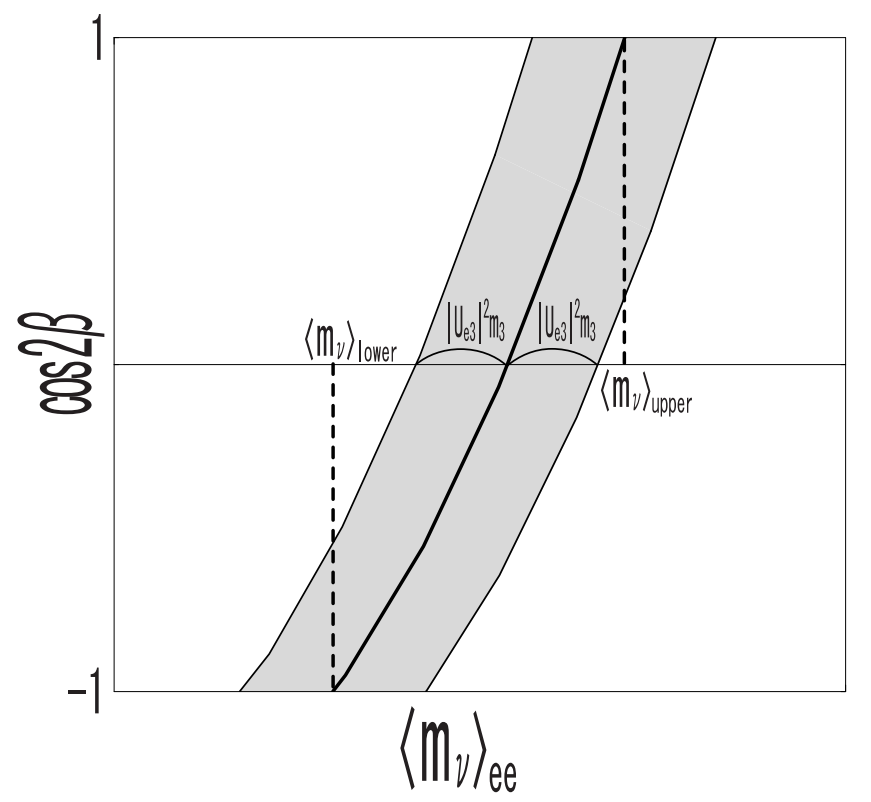

FIG.6 The relation between $\cos 2 \beta$ and $\left\langle m_{\nu}\right\rangle_{e e}$ which is obtained from Eq.(4.11). The solid line is for the case, $U_{e 3}=0$. For $U_{e 3} \neq 0$ case, the relation has a band structure (shaded region). Here we define $\left.\left\langle m_{\nu}\right\rangle_{\text {lower }} \equiv\left|m_{1}-\right| U_{e 2}\right|^{2}\left(m_{1}+m_{2}\right) \mid$ and $\left\langle m_{\nu}\right\rangle_{\text {upper }} \equiv m_{1}+\left|U_{e 2}\right|^{2}\left(m_{2}-\right.$ $\left.m_{1}\right)$. 

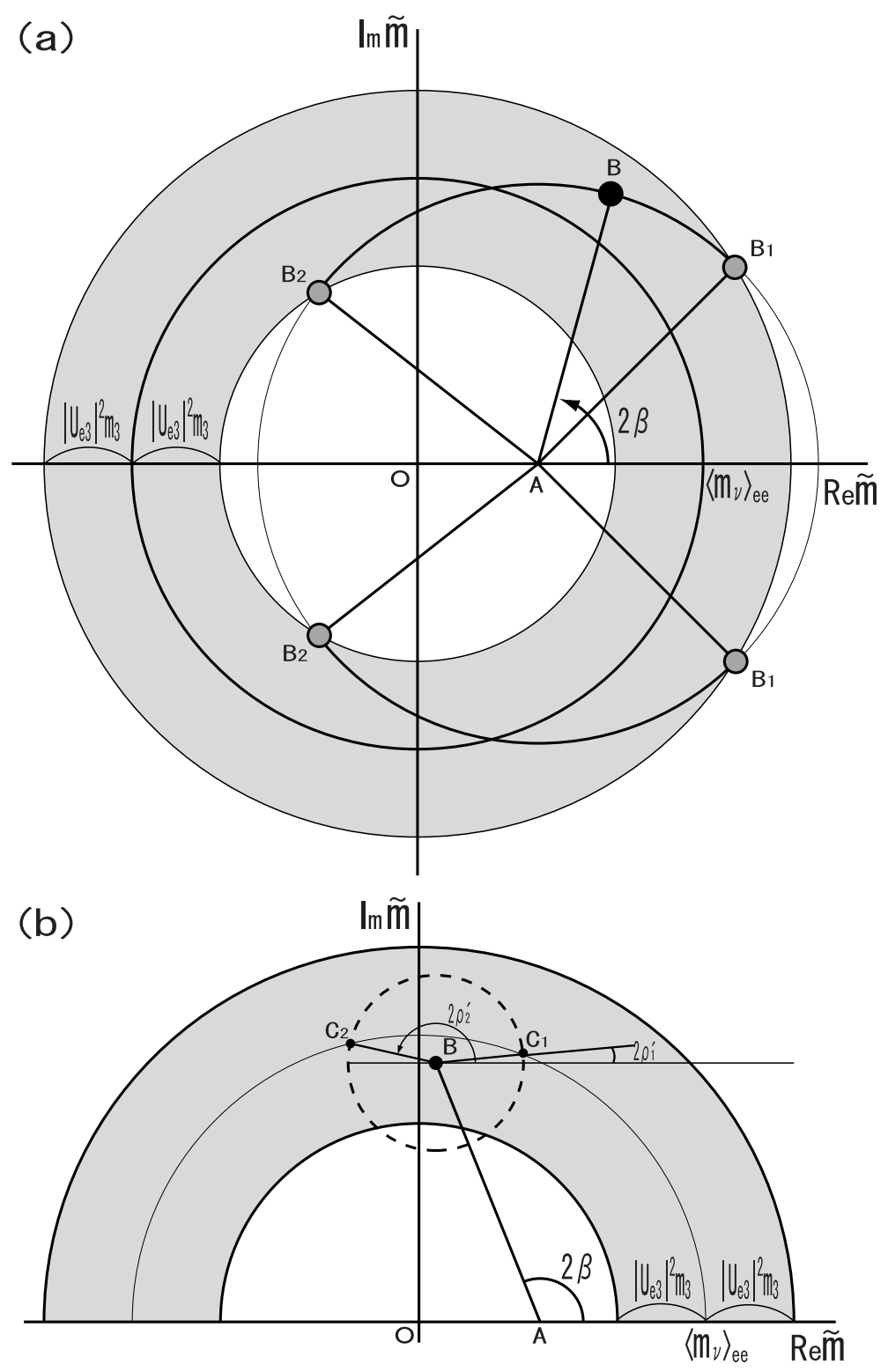

FIG.7 The constraint on $\beta$ and $\rho^{\prime}$ for $\left|U_{e 3}\right|^{2} \neq 0$ in the case(A). (a) The $2 \beta$ is ranging from the argument of $\overrightarrow{A B_{1}}$ (angle between $\overrightarrow{A B_{1}}$ and the horizontal axis) to that of $\overrightarrow{A B_{2}}$. Here $O A=\left|U_{e 1}\right|^{2} m_{1}$ and $|A B|=\left|U_{e 2}\right|^{2} m_{2}$. In this diagram we consider the case where the circle of radius $\left|U_{e 2}\right|^{2} m_{2}$ around $A$ intersects with the circles of radius $\left\langle m_{\nu}\right\rangle_{e e} \pm\left|U_{e 3}\right|^{2} m_{3}$ at $B_{1}$ and $B_{2}$. (b) The $\rho^{\prime}$ has two solution $\rho_{1}^{\prime}$ and $\rho_{2}^{\prime}$ for fixed $2 \beta$ since $\overrightarrow{O A}+\overrightarrow{A B}+\overrightarrow{B C}=M_{e e}$. The dotted line is the circle of radius $\left|U_{e 3}\right|^{2} m_{3}$ around $B$ which intersects the circle of radius $\left\langle m_{\nu}\right\rangle_{e e}$ around the origin at $C_{1}$ and $C_{2}$. Here we refer the point $\left|U_{e 1}\right|^{2} \widetilde{m_{1}}+\left|U_{e 2}\right|^{2} \widetilde{m_{2}}$ as $B$. 


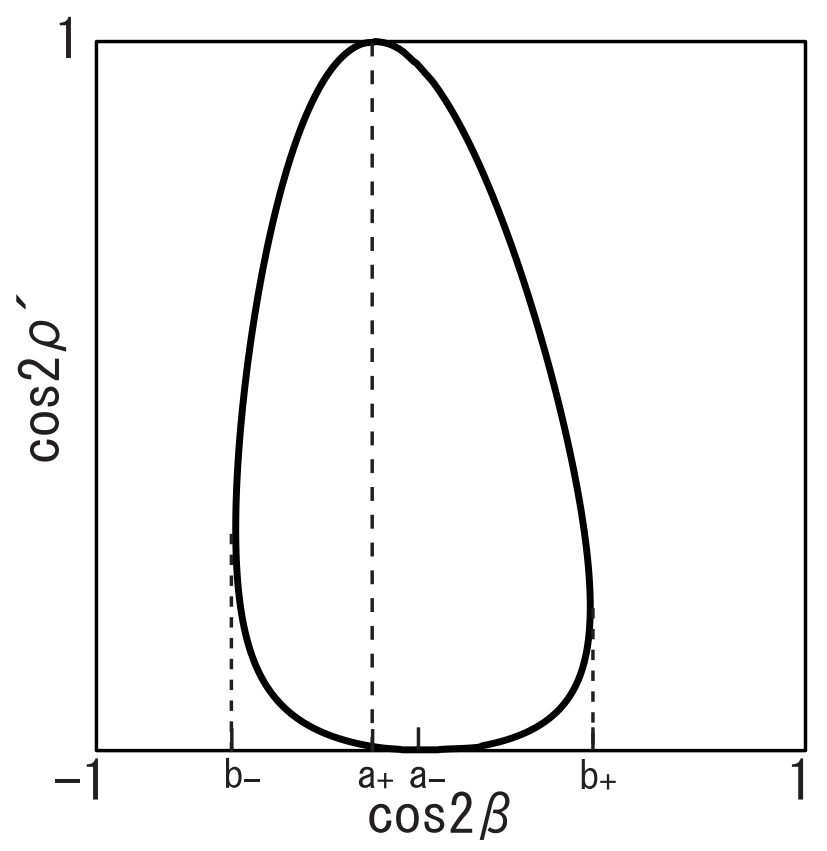

FIG.8 The relation between $\cos 2 \beta$ and $\cos 2 \rho^{\prime}$. The $2 \rho^{\prime}$ has always two solutions for fixed $\beta$ as shown in Fig. 7(b). The $a_{ \pm}$and $b_{ \pm}$are given by $a_{ \pm} \equiv \frac{\left\langle m_{\nu}\right\rangle_{e e}^{2}-\left(\left|U_{e 1}\right|^{2} m_{1} \pm\left|U_{e 3}\right|^{2} m_{3}\right)^{2}-\left|U_{e e}\right|^{4} m_{2}^{2}}{2\left|U_{e 2}\right|^{2} m_{2}\left(\left|U_{e 1}\right|^{2} m_{1} \pm\left|U_{e 3}\right|^{2} m_{3}\right)}$ and $b_{ \pm} \equiv \frac{\left(\left\langle m_{\nu}\right\rangle_{e e} \pm\left|U_{e}\right|^{2} m_{3}\right)^{2}-\left|U_{e l}\right|^{4} m_{1}^{2}-\left|U_{e 2}\right|^{4} m_{2}^{2}}{2\left|U_{e 1}\right|^{2}\left|U_{e 2}\right|^{2} m_{1} m_{2}}$.

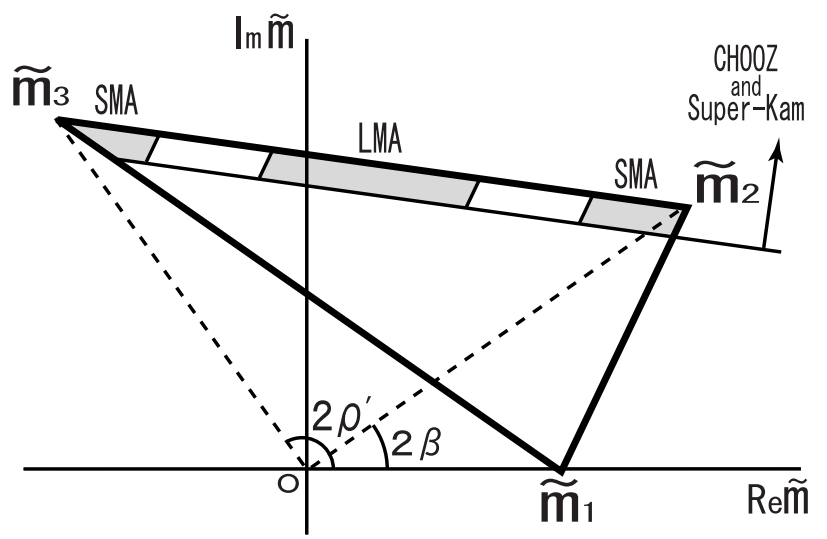

FIG.9 The allowed region of $M_{e e}$ for the case (B) from the CHOOZ and the Super Kamiokande experiments. The position of $M_{e e}$ is restricted in the shaded area which is in the neighborhood of the edge $\widetilde{m_{2}} \widetilde{m_{3}}$. 